\title{
Ética em pesquisa: avanços e desafios
}

DOI: $10.3395 /$ reciis.v2.Sup1.212pt

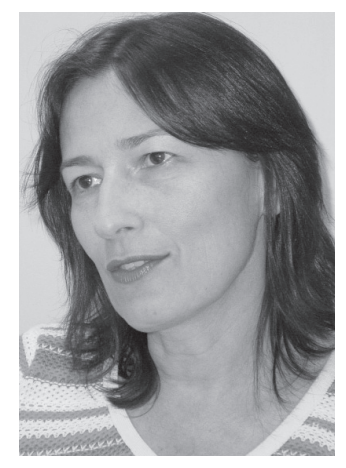

Dirce Guilhem

Departamento de Enfermagem, Universidade de Brasília, Brasília, DF, Brasil guilhem@unb.br

\section{Resumo}

O tema da ética em pesquisa faz parte da agenda científica em distintos campos do conhecimento. A diversidade dos aspectos envolvidos demonstra a complexidade dos dilemas que emergem nesse cenário. Este texto direciona especial atenção a quatro pontos que merecem reflexão, seja no contexto internacional, seja no nacional: o vínculo existente entre método e ética em pesquisas, o processo de treinamento e capacitação de jovens cientistas, a defesa de um único standard para a realização de pesquisas colaborativas internacionais e a proposta de revisão do atual texto da Declaração de Helsinque. A análise desses pontos permite verificar que houve avanços significativos nesse campo, porém a constante tentativa de flexibilizar os requisitos éticos utilizados para nortear a prática científica pode ocasionar um retrocesso que terá sérias conseqüências para os participantes dos estudos. O Brasil é um país privilegiado, pois o sistema brasileiro de revisão ética das pesquisas - o Sistema CEP/Conep - está vinculado ao controle social, o que garante originalidade e legitimidade ao sistema para defender os interesses e direitos das pessoas incluídas nos estudos.

\section{Palavras-chave}

ética em pesquisa; ciência; capacitação; pesquisa; Declaração de Helsinki

O tema da ética em pesquisa, em suas diferentes facetas e nuances, faz parte da agenda científica nas diferentes áreas do conhecimento e demonstra a complexidade das questões que emergem nesse cenário. Aproximar ciência e ética não é uma tarefa simples e o processo de desenvolvimento da sensibilidade ética representa um desafio na atualidade. A incorporação de requisitos éticos no contexto da prática científica tem como objetivo principal garantir a proteção requerida aos participantes dos estudos e fortalecer comportamentos e valores que favoreçam o exercício da equidade, a responsabilidade e o respeito aos direitos humanos.

O Brasil é um país no qual a discussão sobre os princípios éticos que devem nortear a condução de pesquisas avançou rapidamente, o que permitiu a criação e consolidação do sistema brasileiro de revisão ética das pesquisas. Esse sistema foi instituído pela Resolução CNS 196/1996, elaborada e divulgada pelo Conselho 
Nacional de Saúde (Conselho Nacional de Saúde 1996). É denominado Sistema CEP/Conep e está constituído por uma Comissão Nacional de Ética em Pesquisa e por cerca de 581 comitês em funcionamento no País, localizados em universidades, centros de pesquisa e hospitais (Conselho Nacional de Saúde 2008a). A partir de 1996, foram elaboradas resoluções complementares para as pesquisas incluídas nas denominadas áreas temáticas especiais - cooperação estrangeira, reprodução humana, populações indígenas, genética humana, projetos multicêntricos, armazenamento de materiais biológicos -, como forma de contemplar os avanços científicos e os novos dilemas que surgiram nesse período (Conselho Nacional de Saúde 2008b).

Considerando o cenário internacional, e também o nacional, no que se refere à ética em pesquisa, escolhi quatro temas que merecem nossa atenção: o vínculo existente entre método e ética em pesquisas, o processo de treinamento e capacitação de jovens cientistas, a defesa de um único standard para a realização de pesquisas colaborativas internacionais e a proposta de revisão do atual texto da Declaração de Helsinque.

A realização de pesquisas envolvendo seres humanos tem um objetivo principal: produzir conhecimento generalizável, que possa ser incorporado às políticas públicas de saúde, o que demonstrará sua importância social e científica (Emanuel 2000; 2004). Dessa forma, as evidências produzidas poderão contribuir para a melhoria da qualidade de vida das pessoas, grupos e comunidades, para o entendimento dos determinantes sociais do processo saúde-doença, bem como para propor soluções estratégicas capazes de transpor barreiras e melhorar a atenção em saúde pública direcionada à população em geral. Acompanhando essa argumentação, é possível compreender que a reflexão ética deve acompanhar as diferentes fases de desenvolvimento da pesquisa, que se inicia com sua concepção, definição da pergunta de pesquisa, escolha do desenho do estudo, entrada no campo de trabalho e condução da investigação, análise dos dados e divulgação dos resultados (Guilhem \& Zicker 2007).

Diferentemente do que se poderia pensar, o estreito vínculo entre método e ética está presente em todas as diretrizes éticas internacionais utilizadas como referência para subsidiar a realização de pesquisas. O Código de Nurembergue (1947) foi elaborado no pós-guerra e é considerado o primeiro documento internacional a incorporar requisitos éticos para a realização de pesquisas. As salvaguardas protetoras incluem a obtenção do consentimento informado ${ }^{1}$ e a voluntariedade da participação como forma de minimizar os riscos a que os participantes poderiam ser expostos. Paralelamente a isso, o documento dedica seis de seus dez parágrafos para demonstrar a importância da validade científica e social da pesquisa envolvendo seres humanos.

A Declaração de Helsinque, elaborada pela Associação Médica Mundial (AMM), foi um documento inicialmente direcionado à classe médica e tinha como objetivo resgatar a imagem dos médicos-cientistas perante a sociedade (World Medical Association 1964).
De fato, "o documento é um desdobramento de alguns dos preceitos éticos do Código de Nurembergue, porém com objetivos mais concretos de intervenção na prática de pesquisa biomédica" (Guilhem \& Diniz 2008: 21). Em sua primeira revisão, elaborada na $29^{\mathrm{a}}$ Reunião da Associação, realizada em Tóquio no ano de 1975, foi introduzido o conceito de revisão do protocolo de pesquisa por um comitê independente, especializado no tema da ética em pesquisa, como consta no Artigo I.2:

"O desenho e os procedimentos de cada estudo experimental envolvendo seres humanos devem estar claramente formulados em um protocolo de pesquisa que deverá ser submetido para apreciação de um comitê [de ética] independente para considerações, comentários e aconselhamento" (World Medical Association 1975).

O reconhecimento da importância de revisão e reflexão éticas sobre o protocolo experimental levou a AMM a introduzir essa questão na Declaração de Helsinque. Esta se tornou a primeira diretriz a propor esse vínculo como um requisito fundamental para a realização de pesquisas incluindo participantes humanos.

Outro documento que merece ser mencionado é o Relatório Belmont, pois é amplamente utilizado como referência para a revisão ética dos estudos, além de ser utilizado como base para a formulação de outras diretrizes internacionais e nacionais. Três princípios éticos foram definidos como requisitos fundamentais para nortear a realização de pesquisas: respeito pelas pessoas, beneficência e justiça. Cada um deles assume correspondência prática: consentimento informado, avaliação entre riscos e benefícios e seleção equitativa dos sujeitos de pesquisa, elementos fundamentais que apontam para a importância do vínculo entre ciência e ética, o que está expresso nesta passagem do texto:

“(...) a avaliação apresenta tanto uma oportunidade quanto uma responsabilidade de coletar informações sistemáticas e abrangentes sobre a pesquisa proposta. Para o pesquisador, é um meio de examinar se esta investigação está adequadamente delineada. Para um comitê de ética, representa um método para determinar se os riscos que serão apresentados aos sujeitos são justificados. Para os sujeitos em potencial, a avaliação auxilia na decisão entre participar ou não" [sem grifos no original] (Diniz 2008: 198).

As demais diretrizes que se seguiram utilizaram esses três documentos citados anteriormente como referência para sua elaboração e incorporaram seus princípios e orientações. Isso ocorreu, também, com a Resolução CNS 196/1996, que aponta para a necessidade de se realizar uma avaliação ética da abordagem metodológica (Conselho Nacional de Saúde 1996). O capítulo III, intitulado Aspectos Éticos da Pesquisa Envolvendo Seres Humanos, delimita os requisitos que devem ser analisados para comprovar a eticidade de uma pesquisa. Essa concepção é importante, pois é preciso compreender que a metodologia escolhida, envolve procedimentos variados e que podem ter implicações éticas para os participantes.

No entanto, não cabe ao comitê de ética em pesquisa julgar a escolha metodológica do pesquisador. Essa é 
uma atividade que deve ser desenvolvida por seus pares, uma vez que o referencial científico é determinado pelos diferentes campos do conhecimento. Porém, é responsabilidade do comitê avaliar a consistência dos procedimentos e das técnicas de análise dos dados indicados, para verificar se os objetivos propostos podem ser alcançados, a pergunta de pesquisa respondida e se existe garantia de proteção para os participantes. Esse processo é de fundamental importância, pois um estudo cientificamente questionável, com procedimentos inconsistentes, revelará uma falha ética e poderá colocar os sujeitos do estudo sob riscos adicionais, além de ocasionar gastos de tempo e de recursos financeiros e humanos, com resultados que não poderão ser aproveitados (Loue 2002).

Essas considerações nos levam ao segundo tema proposto: o processo de capacitação ética de jovens cientistas. Novamente, todos os documentos internacionais e a regulamentação brasileira apontam para a necessidade de capacitação técnica dos pesquisadores, o que deverá ser comprovado por meio de seu curriculum vitae. Contudo, nem sempre esse preparo é acompanhado pela reflexão e aprendizado de valores e comportamentos que permitam o desenvolvimento da sensibilidade ética durante o processo de formação acadêmica. Essa compreensão assume relevância especial, pois a eticidade requerida para a condução de pesquisas envolvendo seres humanos, “(...) ultrapassa o processo de revisão dos protocolos efetuado pelos comitês de ética em pesquisa" e implica na adoção de posturas responsáveis por parte dos investigadores (Zicker 2006: 2).

Existe consenso sobre o fato de que os princípios éticos utilizados para direcionar a prática de pesquisa sejam considerados universais. Porém, a depender do local onde as pesquisas são realizadas - países em desenvolvimento -, existem várias questões que permeiam esse cenário e que assumem papel relevante para possíveis desvios na condução dos estudos. Entre eles podem ser citados: a falta de acesso ou acesso limitado a serviços de saúde e educação, os padrões de cuidado oferecidos pelos serviços locais de saúde - que estão longe de serem aqueles praticados por países desenvolvidos, a falta de acesso a bens de consumo e a própria lacuna no treinamento dos pesquisadores. Fica evidente, portanto, que a “(...) existência de documentos internacionais e de regulamentação nacional representa o passo inicial para a adoção de princípios éticos na prática de pesquisa" (Zicker 2006: 1).

Essa é uma reflexão que precisa ser ampliada quando nos deparamos com situações constrangedoras relacionadas a comportamentos adotados por pesquisadores durante a realização das investigações. A revista Nature publicou recentemente artigos que demonstravam postura questionável de investigadores estadunidenses no processo de condução dos estudos. Sob o título de Scientists behaving badly, os autores apresentaram o resultado de um estudo realizado com 3247 pesquisadores, entre os quais 1479 eram jovens cientistas e 1768 estavam no meio de suas carreiras (Martison 2005). Cerca de 35\% dos entrevistados mencionaram haver adotado, nos últi- mos três anos, algum tipo de conduta imprópria em sua atividade. Entre eles, os comportamentos mais citados foram: falsificação, fabricação e ocultamento de dados, plágio, quebra da confidencialidade, desconsideração pelo bem-estar dos participantes, utilização das idéias de outras pessoas sem solicitar permissão e modificação do desenho, da metodologia e dos resultados dos estudos em obediência à pressão por parte dos financiadores. Um aspecto que apresentou diferença estatisticamente significativa foi o fato de que pesquisadores em início de carreira adotam menor quantidade de comportamentos abusivos que seus colegas na fase intermediária da carreira. Esses resultados apontam para a importância fundamental que assume uma aproximação precoce aos temas correlacionados aos aspectos éticos requeridos para a condução das pesquisas.

Ainda no contexto estadunidense, outro estudo apontou que muitos incidentes de conduta imprópria em pesquisa não são comunicados às autoridades competentes (Titus 2008). Embora os autores reconheçam as limitações do estudo, os principais comportamentos relatados foram falsificação ou fabricação de dados e plágio. Uma vez mais, os pesquisadores seniores ou em meio de carreira foram os que mais adotaram esses tipos de comportamentos. Esses estudos demonstram que essa prática existe, porém não pode ser considerada predominante no contexto da realização de pesquisas. Ainda assim, é preciso ter cautela, pois “(...) a fraude científica vem crescendo em função da grande quantidade de dinheiro envolvida em pesquisa", a necessidade de captar recursos para as instituições onde os cientistas trabalham, a competitividade existente entre os pesquisadores e, ainda, o medo de perder o emprego (Goliszek 2004: 44).

Mas essa não é uma prerrogativa apenas de cientistas de países desenvolvidos. Apenas como exemplo, no estudo coordenado por David M. Kent, os autores apresentavam um hipotético de pesquisa com terapia para HIV/Aids (Kent 2003). Verificou-se que 86\% dos entrevistados aceitariam conduzir uma pesquisa em que a “... terapia testada tivesse benefício potencial local, mesmo quando essa terapia fosse reconhecidamente inferior àquelas utilizadas no país que patrocina a pesquisa...”. Ou seja, a adoção de um duplo standard no desenvolvimento das pesquisas é uma das possibilidades nesse cenário, independente do local onde o pesquisador se encontre. Esses resultados demonstram certo desalento, principalmente porque os investigadores entrevistados haviam recebido alguma forma de treinamento relacionado à ética em pesquisa.

A incorporação de valores e a aquisição de competência ética é um processo que exige reflexão e deve ser iniciado já nas primeiras etapas do processo de formação. A utilização de metodologias ativas de ensino-aprendizagem pode contribuir para a capacitação dos futuros pesquisadores e prepará-los para enfrentar e minimizar possíveis conflitos de interesses que possam emergir no cotidiano da prática científica.

Esses exemplos nos permitem iniciar o terceiro tema: a defesa de um único standard para o desenvolvimento 
de pesquisas colaborativas internacionais, também denominadas estudos multicêntricos internacionais (Meinert 1996). Este tipo de pesquisa é conduzido em diferentes contextos, marcados por extrema desigualdade, e sua realização suscita grandes questionamentos.

Tradicionalmente, as pesquisas multicêntricas internacionais obedecem à seguinte lógica: a) são financiadas por instituições localizadas em países desenvolvidos: universidades, indústrias farmacêuticas, agências governamentais ou organismos internacionais - os denominados países patrocinadores -, e realizadas em países em desenvolvimento - os países anfitriões (Guilhem \& Diniz 2008); b) essas instituições são responsáveis por delinear o estudo, escolher os procedimentos e o processo de randomização da amostra nos diferentes braços, ou seja, de definir quais serão os participantes que serão incluídos no(s) grupo(s) experimental(is) ou no grupo controle, além de selecionar os pesquisadores e centros de pesquisa onde o estudo será conduzido; c) todos os centros de pesquisa seguem um mesmo protocolo de pesquisa em todos os centros participantes, independente de sua localização; d) as instituições patrocinadoras são, também, as proprietárias dos dados coletados, que serão sistematizados no país de origem da pesquisa, o país onde está localizado o patrocinador da pesquisa; e) a divulgação dos resultados é realizada pelo patrocinador e qualquer publicação sobre parte do dados efetuada por um dos membros da equipe deverá obter a autorização formal do patrocinador (Guilhem 2003). Há, portanto, uma hierarquização nesse processo, e é preciso considerar quem serão os grandes beneficiados quando da utilização dos resultados encontrados.

O que caracteriza o duplo standard é a adoção de um duplo padrão de tratamento durante o processo de realização da pesquisa, escolhido em função do país onde se realiza a pesquisa e dos participantes que integrarão o estudo. Essa não é a posição da atual versão da Declaração de Helsinque, que, em seu parágrafo 29, define que qualquer pesquisa somente poderá ser realizada se obedecer ao seguinte critério:

"Os benefícios, riscos, encargos e eficácia de um novo método devem ser testados comparativamente com os melhores métodos atuais profiláticos, diagnósticos e terapêuticos existentes. Isso não inclui o uso de placebo ou de não-tratamento em estudo em que não existam métodos profiláticos, diagnósticos ou terapêuticos comprovados" (World Medical Association 2000: 4).

Para aqueles que defendem o duplo standard, porém, a realização de estudos controlados por placebo, mesmo na presença de tratamentos internacionalmente consolidados, seria aceitável se esse tratamento não estiver disponível no país anfitrião. Ou seja, a desigualdade global, que permite a adoção de diferentes padrões de cuidado entre os países, seria uma situação plenamente justificável para a utilização do duplo standard.

De acordo com a argumentação de Macklin (2004), parte da discussão sobre esse tema teve origem quando se tentou responder a duas perguntas: a) as pesquisas biomédicas deveriam ser conduzidas em países do Terceiro
Mundo quando elas poderiam ser perfeitamente realizadas nos Estados Unidos ou na Europa?; b) é aceitável que os padrões éticos adotados em países industrializados sejam modificados ou flexibilizados quando os mesmos realizam pesquisas em países em desenvolvimento ou, ainda, em países pobres? Uma possível resposta à primeira pergunta é a de que há um grande número de pesquisas multicêntricas sendo conduzidas em países desenvolvidos. Basta entrar em plataformas de registro de ensaios clínicos e verificar a localização dos estudos (US National Institutes of Health 2008). Mas é preciso considerar que, nesses países, existem critérios muito rígidos para a condução dos estudos, e organismos de proteção aos participantes estão formalmente constituídos. A resposta à segunda pergunta origina a grande controvérsia, já que a modificação dos padrões adotados entre países ricos e pobres gera o duplo standard em pesquisa, uma situação eticamente inaceitável.

A defesa de um único standard em pesquisa tem como ponto de partida a Declaração de Helsinque e a correlação existente com artigos de outras diretrizes internacionais, principalmente aquelas destinadas à realização de pesquisas nos países em desenvolvimento. Porém, a presença desses documentos nem sempre é capaz de transcender ao circuito de fragilidades a que estão expostas pessoas e países em um contexto econômico e social extremamente desigual. Ainda na concepção de Macklin (2004), é possível realizar investigações multinacionais e, ao mesmo tempo, respeitar e proteger a dignidade dos participantes incluídos nos estudos. Mas, para isso, seria necessário adotar a cultura de direitos humanos, utilizando-a como referência e colocando-a acima dos documentos existentes, o que deveria ser feito de fato por países, comunidades e, por que não, pelas empresas e instituições que detêm o poder econômico para a condução das pesquisas.

Nos últimos dois processos de revisão da Declaração de Helsinque, a postura brasileira foi a de se opor a qualquer mudança na declaração que permitisse a emergência do duplo standard, defendendo a utilização de valores e princípios éticos universais no processo de realização das pesquisas. Isso representa a defesa incondicional de um único standard e a luta pela manutenção de garantias e direitos dos participantes.

Esse debate nos leva ao último tema proposto: a proposta de revisão do atual texto da Declaração de Helsinque. O processo iniciou-se no mês de maio de 2007, quando Associações Médicas Nacionais e os diferentes atores envolvidos na realização de pesquisas médicas foram convidados a identificar parágrafos da Declaração que requeriam revisão, propostas específicas de esclarecimento ou, ainda, novos tópicos que deveriam ser incluídos no documento. A partir de então, foram elaboradas duas propostas iniciais, apresentadas para a comunidade científica e colocadas sob consulta pública. A partir da inclusão e discussão dos tópicos propostos, a versão final será discutida e votada na próxima assembléia da AMM, a ser realizada em Seul - Coréia do Sul, em outubro de 2008. Vale analisar, portanto, alguns dos 
pontos que estão em discussão e que, se forem aprovados tal como estão, poderão flexibilizar as garantias e proteções dirigidas atualmente aos participantes.

Entre inúmeros questionamentos, são três os pontos que ocasionam maior controvérsia no novo texto proposto. $\mathrm{O}$ primeiro deles diz respeito à utilização de placebo. Foi incluído um desdobramento ao parágrafo 32, que diz o seguinte:

"Quando, por razões metodológicas convincentes e cientificamente sólidas, for necessário o uso de placebo a fim de determinar a eficácia ou segurança de um método e os pacientes que receberem o placebo, ou não receberem nenhum tratamento, não estejam sujeitos a qualquer risco adicional de danos graves e irreversíveis" (World Medical Association 2008: 2).

Essa passagem abre caminho para a utilização de placebo mesmo quando existir tratamento internacionalmente consolidado. É preciso questionar em que condições seria eticamente aceitável manter os participantes dos estudos apenas com placebo, privando-os de medicamentos de eficácia comprovada para sua situação de saúde. A defesa aqui é pela manutenção de que o tratamento que está sendo estudado deve ser comparado com a terapia já existente.

Outro aspecto relevante está relacionado ao acesso dos participantes aos medicamentos e tratamentos considerados exitosos pelo estudo. Várias questões emergem nesse contexto: até quando deve ser mantido o fornecimento dos medicamentos após a finalização do estudo? Quem deve assumir a responsabilidade ética e legal de fornecer os medicamentos ao final da pesquisa? O que fazer se o medicamento não está disponível comercialmente ou não for incorporado ao rol de tratamentos fornecido pelo Sistema Único de Saúde? Com exceção da última questão, a resposta a estes questionamentos foi incluída no parágrafo 14, com a seguinte redação:

“(...) O protocolo deve incluir informações quanto ao financiamento, patrocinadores, afiliações institucionais, outros potenciais conflitos de interesse, incentivos aos sujeitos e cláusulas para tratar e/ou compensar os sujeitos que sofrem danos como conseqüência da participação no estudo. O protocolo deve descrever os acordos para o acesso pós-estudo por parte dos sujeitos a métodos identificados como benéficos no estudo ou acesso a outros cuidados ou benefícios apropriados" [sem grifos no original]. (World Medical Association 2008: 1).

$\mathrm{Na}$ verdade, esta é uma modificação semântica sutil, que flexibiliza o acesso anteriormente concedido no parágrafo 30 da versão atual da resolução, onde está indicado que "na conclusão do estudo, todo paciente nele incluído deve ter o acesso assegurado aos melhores métodos profiláticos, diagnóstico e terapêuticos comprovados, identificados pelo estudo" [sem grifos no original] (World Medical Association 2000: 5). Ou seja, garantir o acesso pós-estudo a algo menos do que os melhores métodos existentes é considerado eticamente questionável.

Um último ponto sinaliza para as questões relacionadas à inclusão de crianças e adolescentes como participantes dos estudos. Apesar de serem considerados grupos vulneráveis, existem drogas e tratamentos que poderiam ser benéficos para esses grupos, mas para os quais simplesmente não foi desenvolvido um protocolo específico. A realização de pesquisas com populações vulneráveis deve considerar os benefícios e os conhecimentos que poderão ser produzidos e aplicados para melhorar a qualidade de vida desses grupos. Por isso, é importante que esses estudos sejam conduzidos, certificando-se de que os mesmos obedecem a critérios científicos e éticos para que crianças e adolescentes estejam protegidos. Além disso, um ponto adicional diz respeito ao fato de que sua decisão em participar ou não do estudo deve ser respeitada.

É interessante, ainda, salientar que, embora a Declaração de Helsinque tenha sido incorporada pela comunidade científica de diferentes campos do conhecimento como um documento de referência para o desenvolvimento de pesquisas, ela era um documento dirigido especificamente à classe médica. No entanto, verifica-se, nessa nova versão, a inclusão de parágrafo n.2, no qual a “(...) Associação Médica Mundial convida outros participantes de pesquisas médicas que envolvem seres humanos a adotar esses princípios" (World Medical Association 2008: 1). O reconhecimento da multidisciplinaridade no contexto da pesquisa em saúde pode ser considerado um avanço e o convite à pluralidade de opiniões, o que ampliará o escopo das discussões e contribuirá para garantir a proteção dos participantes das pesquisas

Para finalizar, a análise desses quatro pontos nos permite verificar que houve avanços significativos no diálogo sobre os grandes dilemas que permeiam o desenvolvimento das pesquisas. Entre eles podem ser citados: a delimitação de princípios éticos universais para serem utilizados nesse cenário; a elaboração de documentos internacionais e de legislação nacional; a preocupação dos cientistas, e da sociedade em geral, de que o controle da prática científica não deve estar restrito aos cientistas. $\mathrm{E}$, nesse aspecto, o Brasil conseguiu um grande avanço: o Sistema CEP/Conep está vinculado ao Conselho Nacional de Saúde, instância do controle social, o que garante originalidade ao Sistema Brasileiro de Revisão Ética das Pesquisas e legitimidade para defender os interesses e os direitos das pessoas incluídas nos estudos. O que nos permite defender de forma incondicional a utilização de um único standard na realização de pesquisas conduzidas no país.

\section{Nota}

1. A denominação consentimento informado é utilizada no contexto internacional para designar o processo de obtenção da aceitação voluntária do participante que será incluído no estudo. No Brasil, a terminologia adotada é Termo de Consentimento Livre e Esclarecido. Optei por manter a primeira denominação para os documentos internacionais citados no texto. 


\section{Referências bibliográficas}

Código de Nurembergue. 1947. Disponível em: http:// www.ufrgs.br/bioetica/nuremcod.htm. Acesso em: 3 maio 2008.

Conselho Nacional de Saúde. Resolução n.196, de 10 de outubro de 1996. Diretrizes e Normas Regulamentadoras de Pesquisas Envolvendo Seres Humanos. Brasília: CNS; 1996.

Conselho Nacional de Saúde. Comissão Nacional de Ética em Pesquisa. Boletim Informativo do $1^{\circ}$ Semestre de 2008. Disponível em: http://conselho.saude.gov. br/Web_comissoes/conep/ arquivos/ CONEP INFORMA_jul08.doc. Acesso em: 10 out. 2008.

Conselho Nacional de Saúde. Comissão Nacional de Ética em Pesquisa. Resoluções. Disponível em: http:// conselho.saude.gov.br/Web_comissoes/conep/aquivos/resolucoes/resolucoes.htm. Acesso em: 10 out. 2008.

Diniz D, Guilhem D, Schuklenk U. Ética em pesquisa: a experiência de treinamento de países sul-africanos. Brasília: LetrasLivres; Editora UnB; 2008. p.181-204.

Emanuel EJ. What makes clinical research ethical? J Am Med Assoc. 2000; 283:2701-11.

Emanuel EJ. What makes clinical research in developing countries ethical? The benchmarks of ethical research. J Infect Dis. 2004; 189:930-7.

Golisek A. Cobaias humanas: a história secreta do sofrimento provocado em nome da ciência. Rio de Janeiro: Ediouro; 2004.

Guilhem D. Pesquisas biomédicas multinacionales: ¿es posible mantener un unico estándar desde el escenario de un país en desarrollo? Perspectivas Bioéticas. 2003; 8(15):44-66.

Guilhem D, Diniz D. O que e ética em pesquisa. São Paulo: Brasiliense; 2008.

Guilhem D, Zicker F. Introdução. In: Guilhem D, Zicker F, editores. Ética na pesquisa em saúde: avanços e desafios. Brasília: LetrasLivres; Editora UnB; 2007. p.13-28.

Kent DM. Testing therapies less effective than the best current standard: ethical beliefs in an international sample of researchers. Am J Bioethics. 2003; 3(2):W28W33. Disponível em: http://www.bioethics.net/journal/ j_articles.php?aid=88. Acesso em: 10 out. 2008 .

Loue S. Textbook of research ethics: theory and practice. United States: Kluwer Academic Publishers; 2002.

Macklin R. Double standards in medical research in developing countries. Cambridge: Cambridge University Press; 2004.

Martinson BC. Scientists behaving badly. Nature. 2005; 435(9):437-8.

Meinert CL. Clinical trials dictionary: terminology and usage recommendations. Baltimore: The Johns Hopkins Center for Clinical Trials; 1996.

National Comission for the Protection of Human Subjects of Bioemdical and Behavioral Research (USA). The Belmont Report: ethical principles and guidelines for the protection of the human subjects of research. In: Reich, W.Y. (Org.). Encyclopedia of bioethics. New York: Macmillan; 1995. p.2767-73.

Titus SL. Repairing research integrity. Nature. 2008; 453(19):980-2.

US National Institutes of Health. Clinical Trials: Studies on map; 2008. Disponível em: http://clinicaltrials. gov/ct2/search/map. Acesso em: 12 out. 2008.

World Medical Association. Declaration of Helsinki. Helsinki: WMA; 1964.

World Medical Association. Declaration of Helsinki. Tokyo: WMA; 1975. Disponível em: http://ethics.iit. edu/codes/coe/world.med.assoc.helsinki.1975.html. Acesso em: 10 out. 2008.

World Medical Association. Declaration of Helsinki. Edinburgh: WMA; 2000. p.1-5. Disponível em: http:// www.wma.net/e/policy/pdf/17c.pdf. Acesso em: 12 out. 2008.

World Medical Association. Declaration of Helsinki. Final draft. Geneva: WMA, 2008.

Zicker F. Apresentação. In: Zicker, F. et al. (editores). Bioética e pesquisa com seres humanos: programas acadêmicos e de extensão. Brasília: LetrasLivres; 2006. p.1-8. [CD ROM]. 


\section{Sobre o autor}

\section{Dirce Guilhem}

Dirce Guilhem é graduada em Enfermagem pela Faculdade Adventista de Enfermagem em São Paulo, especialista em Administração Hospitalar (UERJ, 1982) e Enfermagem do Trabalho (Universidade Gama Filho, 1986). Concluiu o mestrado em Psicologia Social e do Trabalho em 1994, e o doutorado em Ciências da Saúde - Bioética em 2000, ambos cursados na Universidade de Brasília. Realizou o Pós-Doutorado em Bioética e Pesquisa com Seres Humanos na Facultad Latinoamerica de Ciencias Sociales - FLACSO em Buenos Aires, no ano de 2003. É Professora Titular da Universidade de Brasília, docente do Curso de Enfermagem e Obstetrícia, orientadora do Programa de Pós-Graduação em Ciências da Saúde e coordenadora do Grupo de Pesquisa: Ética em Pesquisa. Desenvolve pesquisas na área de bioética, gênero, ética em pesquisa, políticas públicas e HIV/Aids. É membro da Comissão Nacional de Ética em Pesquisa - Conep/MS, presidente do Foro Latinoamericano de Comités de Ética en Investigación en Salud e Consultora do Ministério da Saúde - Decit/MS para o tema da Ética em Pesquisa. 